\title{
Circulating Antigens Levels in Different Clinical Forms of the Schistosoma mansoni Infection
}

\author{
Yerkes Pereira e Silva, Evan Secor*, Marcela Orsine Andrade, Naftale Katz, \\ Ana Rabello/ ${ }^{+}$
}

Laboratório de Esquistossomose, Centro de Pesquisas René Rachou-Fiocruz, Av. Augusto de Lima 1715, 30190002 Belo Horizonte, MG, Brasil *Division of Parasitic Diseases, Laboratory of Parasitology, Centers for Disease Control and Prevention, Atlanta, GA, USA

With the aim to evaluate the circulating cathodic antigen (CCA) levels in relation to the different clinical phases of Schistosoma sp. infection a sandwich ELISA using monoclonal antibody $5 H 11$ was performed. The sera of three groups of 25 Brazilian patients with acute, intestinal and hepatosplenic forms of S. mansoni infection were tested and compared to a non-infected control group. Patients and control groups were matched for age and sex and the number of eggs per gram of feces was equally distributed among the three patient groups. Sensitivity of 100\%, 72\%, 52\% of the assay was observed for the intestinal, hepatosplenic and acute toxemic groups respectively. The specificity was $100 \%$. Intestinal and hepatosplenic groups presented CCA levels significantly higher in comparison to those observed for acute patients ( $F$-ratio $=2,524 ; p=0.000$ and F-ratio $=6,314 ; p=0.015$ respectively). There was no significant difference of CCA serum levels between hepatosplenic and intestinal groups $($ F-ratio $=1,026 ; p=0.316)$.

Key words: Schistosoma mansoni - circulating antigens - diagnosis - ELISA

Presence of adult worm antigens in serum and urine of mice and hamsters infected with Schistosoma mansoni was first reported in 1967 (Berggren $\&$ Weller 1967). Two main types of circulating antigens (CA) were described. The circulating anodic antigen (CAA), negatively charged proteoglycan and the circulating cathodic antigen (CCA), a polysaccharide with a cathodic migration (Deelder et al. 1976). Quantification of CAA and CCA in serum and urine has shown to be a valuable tool for the diagnosis of active Schistosoma sp. infection in endemic areas (Deelder et al. 1989b, 1994, De Jonge et al. 1989, Van Lieshout et al. 1992). Levels of these CA are highly correlated with intensity of infection and a rapid clearance after successful chemotherapy has been demonstrated (Van Lieshout et al. 1993, Agnew et al. 1995, Van't Wout et al. 1995).

A high sensitivity for CAA (using Mab 1201B10-A) and CCA (using Mab 54-5C10-A) detection was observed using sera of selected Brazilian patients. Significantly higher CCA levels were found in patients with hepatosplenomegaly

\footnotetext{
This work was partially supported by Fapemig and Brazilian National Research Council (CNPq).

${ }^{+}$Corresponding author. Fax: +55-31-295.3115 E-mail: ana@netra.cpqrr.fiocruz.br

Received 22 May 1998

Accepted 28 August 1998
}

as compared to patients with intestinal schistosomiasis, where as CAA levels of patients with these clinical forms did not show significant differences (De Jonge et al. 1991).

The Mab 5H11 CCA sandwich enzyme linked immunosorbent assay - ELISA is a sensitive and specific method for the detection of active schistosomiasis mansoni and an useful tool for monitoring specific chemotherapy (Barsoum et al. 1990). Schistosoma negative control groups that presented other helminthic infections i.e. Ascaris lumbricoides, hookworm, Strongyloides stercoralis, Onchocerca volvulus, Dipetalonema perstans, Trichuris and Loa loa were negative in the assay (De Jonge et al. 1990). This assay has also been used in mice to evaluate vaccine-induced resistance against $S$. mansoni infection (Barsoum et al. 1991).

Clinical acute schistosomiasis may commonly develop in the immunologically naive vertebrate host (Rabello 1995). Six to eight weeks after the initial contact with water infested by schistosome cercariae, infected patients present fever, toxemia, weakness, weight loss, diarrhea, abdominal pain, cough, myalgia, arthralgia, urticaria, edema, and enlargement of the liver and spleen. These clinical manifestations vary in intensity from relatively mild to severe and are easily confused with other syndromes with hepatosplenic involvement as infection by Salmonela sp., malaria and hepatitis (Neves 1965, Lambertucci 1993, Rabello 1995, Rabello et al. 1995). 
Recently, two considerable advances were made towards the characterization of acute schistosomiasis. Abdominal ultrasonography showed that liver and spleen enlargement and periportal and peripancreatic lymphadenomegaly are characteristic findings of acute schistosomiasis (Rabello et al. 1994). In addition, specific IgG and IgM antibodies against keyhole limpet haemocyanin (KLH) (Alves Brito et al. 1992) and specific IgA antibodies against soluble egg (SEA) antigens were described as being related to acute schistosomiasis mansoni (Rabello et al. 1995). It has also been shown that in acute schistosomiasis, increased levels of anti-KLH IgM and IgG and IgM and antiSEA IgA antibodies positively correlated with morbidity, allowing for age and levels of water contact (Rabello et al. 1995).

The aim of the present study was to evaluate the presence of CA in serum of acute and chronic (intestinal and hepatosplenic) $S$. mansoni infection compared to a non infected control group.

\section{MATERIALS AND METHODS}

Study population - Table I summarizes four study groups; each group was composed of 25 individuals that were selected in such a way that age and sex were equally distributed among the four groups and infected patients were matched for number of eggs per gram of feces (EPG).

Group 1 - intestinal clinical form - infected patients without hepatosplenomegaly;

Group 2 - hepatosplenic clinical form - infected patients with palpable spleen and liver;

Groups 1 and 2 were selected from endemic areas in the State of Minas Gerais, Brazil.

Group 3 - acute clinical form - patients with the acute clinical form of schistosomiasis. Diag-

TABLE I

Age and number of eggs per gram of feces (EPG) of the four study groups

\begin{tabular}{lcc}
\hline $\begin{array}{l}\text { Group } \\
\text { no. }\end{array}$ & $\begin{array}{c}\text { Age (years })^{a} \\
\text { mean (min-max) }\end{array}$ & $\begin{array}{c}\mathrm{EPG}^{b} \\
\text { geometric mean } \\
\text { (min-max) }\end{array}$ \\
\hline $\begin{array}{l}\text { G 1- Intestinal } \\
25\end{array}$ & $\begin{array}{c}19.4 \\
(7-71)\end{array}$ & $\begin{array}{c}124.8 \\
(12-1362)\end{array}$ \\
G 2 - Hepatosplenic & 23.4 & 103.7 \\
25 & $(6-63)$ & $(12-678)$ \\
G 3 - Acute & 15.4 & 59.6 \\
25 & $(6-42)$ & $\left(0^{c}-2084\right)$ \\
G 4 - Non infected & 21.4 & \\
25 & $(7-48)$ & - \\
\hline
\end{tabular}

$a$ : F-ratio $=1.43 ; \mathrm{p}=0.24 ; b$ : F-ratio $=1.75 ; \mathrm{p}=0.18 ; c$ : zero means that the diagnosis was based on the qualitative sedimentation method. nosis was based on epidemiological and clinical features, after an average of 50 days of water contact, the presence of $S$. mansoni eggs in stools was determined by Kato-Katz method (Katz et al. 1972), or the qualitative sedimentation method (Hoffman et al. 1934) and the presence of high levels of IgG anti-KLH or IgA anti-SEA (Rabello 1995, Rabello et al. 1995).

Group 4 - negative control group - individuals with negative stool examinations from an nonendemic area were selected to serve as negative control group. They were also free of infection with other intestinal worms.

Parasitological examination - Was based on the presence of $S$. mansoni eggs in stools of three fecal samples by the quantitative Kato-Katz method (Katz et al. 1972) or by the qualitative sedimentation method (Hoffman et al. 1934).

Collection and treatment of blood samples Blood samples were collected by venopuncture, and serum was stored at $-20^{\circ} \mathrm{C}$. Samples were treated with trichloro-acetic acid (TCA) to precipitate proteins and/or to dissociate CA from immune complexes (De Jonge et al. 1987). Briefly, equal volumes of each serum sample and 4M TCA were mixed at room temperature for $20 \mathrm{~min}$ and afterwards centrifuged at $14,000 \mathrm{rpm}$ for $15 \mathrm{~min}$. The supernatant was collected and neutralized with an equal volume of $0.25 \mathrm{M}$ sodium carbonate buffer ( $\mathrm{pH}$ 9.6).

Circulating antigen determination - Levels of CCA in serum were determined by antigen capture sandwich ELISA using the monoclonal antibody 5H11/B1 (Barsoum et al. 1990). A standard curve of soluble adult worm antigen preparation (SWAP) was simultaneously assayed on each plate. CCA levels were determined by a linear regression curve, based on this standard curve. The sera were tested in triplicate.

Data analysis - Statistical analysis was performed with SPSS for Windows software. As CCA concentrations and EPG showed skewed distributions and therefore $\log _{10}$ transformed in order to obtain a normal distribution of values. ANOVA was used to compare CCA levels and EPG among groups. To evaluate correlation, Pearson's coefficient was determined. A probability of alpha error less than 5\% was considered to be significant.

\section{RESULTS}

The general sensitivity of the CCA detection for all infected patients was $74.7 \%$. CCA was detected in 25 of the $25(100 \%)$ intestinal patients and in 18 of $25(72 \%)$ hepatosplenic patients. For the acute toxemic group a sensitivity of $52 \%, 13$ of 25 patients, was observed. In the non-infected persons CCA was not detectable, so the specificity was $100 \%$. 
The Figure depicts the CCA levels of the four groups of individuals studied. Levels of CCA were significantly different among the three infected groups $(\mathrm{F}$-value $=8.643 ; \mathrm{p}=0.004)$. Intestinal and hepa-tosplenic groups had higher levels of CCA when compared to the acute patients (F-ratio $=$ 2.524; $\mathrm{p}<0.001)$, (F-ratio $=6.314 ; \mathrm{p}=0.0154)$ respectively. There were no significant differences in CCA levels between the hepatosplenic and the intestinal groups $(\mathrm{F}$-ratio $=1.026 ; \mathrm{p}=0.3160)$.

The correlation coefficients of age with CCA levels, age with EPG and CCA levels with EPG are presented in Table II. Except for the intestinal group among the infected patients, significant correlations were observed between CCA levels and EPG. Correlations between CCA levels and age were observed for intestinal and hepatosplenic groups. A correlation between EPG and age was only observed in the hepatosplenic group. When all patients were considered, a positive correlation between EPG and CCA levels was observed $(\mathrm{r}=0.4625 ; \mathrm{p}<0.001)$.

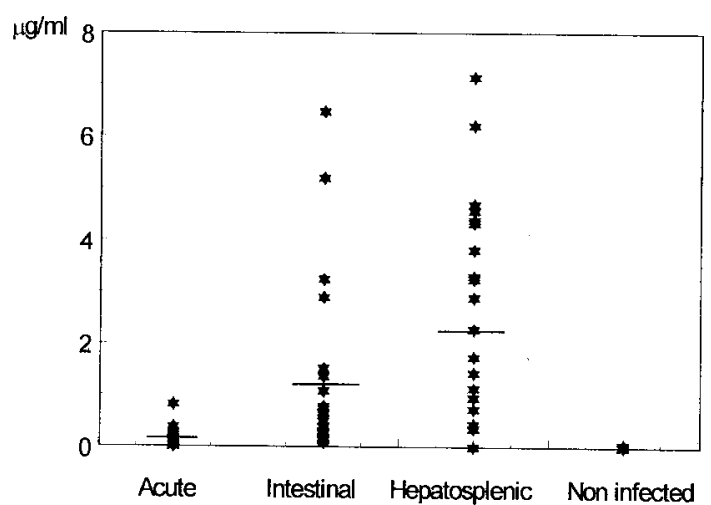

Levels of circulating cathodic antigens of hepastosplenic, intestinal and acute patients with schistosomiais mansoni and non infected group. Each star represents the mean of triplicate sample test results.

\section{DISCUSSION}

The principal conclusion of the present work rests on the observed differences of CCA levels among the different clinical forms of schistosomia- sis mansoni, irrespective to the number of $S$. mansoni per gram of feces. In acute schistosomiasis patients, lower levels of CA were observed compared to patients with intestinal or hepatosplenic clinical forms that had similar levels of egg excretion.

Different values of sensitivity were obtained among the different groups of patients evaluated in this study. This can be considered as a direct consequence of the differences observed for CCA levels among the groups. Although no non-significant differences were observed in EPG eliminated by the different groups, in acute schistosomiasis the sensitivity was lower (52\%). Since there was a strong correlation between EPG and CCA levels in the acute group $(r=0.73)$, patients with non detectable CCA presented the lowest EPG. The same figure, $52 \%$ of sensitivity for CCA detection was obtained in a group of Dutch travelers with acute schistosomiasis (Van Lieshout 1996).

The possible explanation that the infection in acute phase would be too recent to have detectable CA does not seem acceptable in this case, since patients were seen 50 days after water contact. It has been demonstrated in mice that five weeks after exposure CCA can be detected (Agnew et al. 1995).

Variances in the amounts of immune complexes present in sera of the different clinical forms might be another reason for the lower concentrations of CA detectable in acute patients. However, pretreatment of sera with trichloroacetic acid fully dissociates immune complexes (De Jonge et al. 1987).

The results of CCA detection in patients with the hepatosplenic clinical form in this study are in disagreement with a previous study on CA with Brazilian patients where CCA levels were higher in patients with hepatosplenic form compared to patients with intestinal schistosomiasis (De Jonge et al. 1991). Although our data confirm the high levels of CCA in sera from hepatosplenic patients, no difference could be observed between CCA levels of hepatosplenic and intestinal patients. The only difference between the previous and the present was the higher number of EPG in the first study (504 for the hepatosplenic group and 408 for the intestinal group).

\section{TABLE II}

Correlation of number of eggs per gram of feces (EPG) with circulating cathodic antigens (CCA) levels, number of eggs per gram of feces with age and CCA levels with age for the different study groups

\begin{tabular}{|c|c|c|c|c|c|c|}
\hline \multirow[t]{2}{*}{ Groups } & \multicolumn{2}{|c|}{ EPG vs CCA levels } & \multicolumn{2}{|c|}{ EPG vs age } & \multicolumn{2}{|c|}{ CCA levels vs age } \\
\hline & $\mathrm{p}$ & $\mathrm{r}$ & $\mathrm{p}$ & $\mathrm{r}$ & $\mathrm{p}$ & $\mathrm{r}$ \\
\hline Acute & 0.000 & 0.7252 & 0.139 & 0.3045 & 0.787 & -0.0568 \\
\hline Intestinal & 0.139 & -0.3046 & 0.176 & -0.2792 & 0.000 & 0.6571 \\
\hline Hepatosplenic & 0.006 & 0.5377 & 0.018 & -0.4701 & 0.165 & -0.2863 \\
\hline Negative & & & & & 0.250 & -0.2389 \\
\hline
\end{tabular}

p: probability value; $r$ : correlation coefficient. 
A higher level of $\mathrm{CA}$ in patients with hepatosplenic disease has been suggested as consequence of an impairment of the clearance of antigen from immune complexes (De Jonge et al. 1991). It has been demonstrated that persistent high levels of CCA contributes to the development of liver pathology (Feldmeier et al. 1986). It is possible that different kinetics of CA in patients with similar intensities of infection are due to different clinical presentations.

\section{ACKNOWLEDGMENTS}

To Maria Mônica de Aguiar Garcia and Luciana de Gouvêa Viana for their technical assistance.

\section{REFERENCES}

Agnew AM, Fulford AJC, De Jonge N, Krijger FW, Rodriguez-Chacon M, Gutsmann V, Deelder AM 1995. The relationship between worm burden and levels of a circulating antigen (CAA) of five species of Schistosoma in mice. Parasitol 111: 67-76.

Alves-Brito CF, Simpson AJG, Bahia-Oliveira LMG, Rabello ALT, Rocha RS, Lambertucci JR, Gazzinelli G, Katz N, Correa-Oliveira R 1992. Analysis of antikeyhole limpet hemocyanin antibody in Brazilians supports its use for the diagnosis of acute schistosomiasis mansoni. Trans R Soc Trop Med Hyg 86: 53-56.

Barsoum IS, Colley DG, Kamal KA 1990. Schistosoma mansoni: detection of circulating antigens in murine schistosomiasis by antigen-capture sandwich ELISA using a monoclonal antibody. Exp Parasitol 71: 107-113.

Barsoum IS, Kamal KA, Bassily S, Deelder AM, Colley DG 1991. Diagnosis of human schistosomiasis by detection of circulating cathodic antigen with a monoclonal antibody. J Infect Dis 164: 1010-1013.

Berggren WL, Weller TH 1967. Immunoelectrophoretic demonstration of specific circulation antigen in animals infected with Schistosoma mansoni. Am J Trop Med Hyg 16: 606-612.

De Jonge N, Fillié YE, Deelder AM 1987. A simple and rapid treatment (tricloroacetic acid precipitation) of serum samples to prevent non-specific reactions in the immunoassay of a proteoglycan. J Immunol Met 99: 195-197.

De Jonge N, Kremsner PG, Krijger FW, Schommer G, Fillié YE, Kornelis D, Van Zeyl RJM, Van Dam GJ, Feldmeier H, Deelder AM 1991. Detection of the schistosome circulating cathodic antigen by enzzyme immuno-assay using biotinylated monoclonal antibodies. Trans $R$ Soc Trop Med Hyg 84: 815-818.

De Jonge N, Rabello ALT, Krijger F W, Kremsner PG, Rocha RS, Katz N, Deelder AM 1991. Levels of the schistosome circulating anodic and cathodic antigens in serum of schistosomiasis patients from Brazil. Trans $R$ Soc Trop Med Hyg 85: 756-759.

De Jonge N, Fillié YE, Hilberath GW, Krijger FW, Polderman AM, Deelder AM 1989. Presence of the schistosome circulating anodic antigen (CCA) in urine of patients with Schistosoma mansoni or Schistosoma haematobium infections. Am J Trop Med Hyg 41: 563-569.
Deelder AM, De Jonge N, Fillié YE, Konelis D, Helaha D, Qian ZL, De Caluwe P, Polderman AM 1989b. Quantitative determination of circulating antigens in human schistosomiasis mansoni using an indirect hemaglutination assay. Am J Trop Med Hyg 40: 50-54.

Deelder AM, Klappe HTM, Van den Aardweg GJMJ, Van Meerbeke EHEM 1976. Schistosoma mansoni demonstration of two circulating antigens in infected hamsters. Exp Parasitol 40: 189-197.

Deelder AM, Qian ZL, Kremsner PG, Acosta L, Rabello ALT, Enyong P, Simarro PP, Van Etten ECM, Krijger FW, Rotmans JP, Fillié YE, De Jonge N, Agnew AM, an Lieshout L 1994. Quantitative diagnosis of Schistosoma infections by measurement of circulating antigens in serum and urine. Trop Geogr Med 46: 233-238.

Feldmeier H, Nogueira-Queiroz JA, Peixoto-Queiroz MA, Doehring E, Dessaint JP, De Alencar JE, Dafalla AA, Capron A 1986. Detection and quantification of circulating antigen in schistosomiasis by monoclonal antibody. II. The quantification of circulating antigens in human schistosomiasis mansoni and haematobium: relationship to intensity of infection and disease status. Clin Exp Immunol 65: 232-243.

Hoffman VA, Pons JS, Janer JL 1934. Sedimentation concentration method in schistosomiasis mansoni. PR J Pub Health Trop Med 9: 283-298.

Katz N, Chaves A, Pellegrino J 1972. A simple device for quantitative stool thick smear technique in schistosomiasis mansoni. Rev Inst Med Trop São Paulo 14: 397-400.

Lambertucci JR 1993. Acute schistosomiasis: clinical, diagnostic and therapeutic features. Rev Inst Med Trop São Paulo 35: 399-404.

Neves J 1965. Estudo clínico da fase pré-postural da esquistossomose mansoni. Revta Assoc méd Minas Gerais 16: 1-16.

Rabello ALT 1995. Acute human schistosomiasis mansoni. Mem Inst Oswaldo Cruz 90: 277-280.

Rabello ALT, Garcia MMA, Pinto da Silva R, Rocha RS, Chaves A, Katz N 1995. Humoral immune response in acute schistosomiasis mansoni: relation to morbidity. Clin Infect Dis 21: 608-615.

Rabello ALT, Pinto da Silva RA, Rocha RS, Katz N 1994. Abdominal ultrasonography in acute clinical schistosomiasis mansoni. Am J Trop Med Hyg 50: 748-752.

Van Lieshout L, De Jonge N, El Marsy NA, Mansour MM, Krijger FW, Deelder AM 1992. Improved diagnostic performance of the circulating antigen assay in human schistosomiasis by parallel testing for circulating anodic and cathodic antigens in serum and urine. Amer J Trop Med Hyg 47: 463-469.

Van Lieshout L, De Jonge N, Mansour MM, Bassily S, Krijger FW, Deelder AM 1993. Circulating cathodic antigens levels in serum and urine of schistosomiasis patients before and after chemotherapy with praziquantel. Trans R Soc Trop Med Hyg 87: 311-312.

Van't Wout AB, De Jonge N, Wood SM, Van Lieshout L, Mitchell GF, Deelder AM 1995. Serum levels of circulating anodic antigen and circulating cathodic antigen detected in mice infected with Schistosoma japonicum or Schistosoma mansoni. Parasitol Res 81: 434-437. 